\title{
Characterizing the Role of TaWRKY13 in Salt Tolerance
}

\author{
Shuo Zhou ${ }^{1,+}+{ }^{+}$, Wei-Jun Zheng ${ }^{2,+}$, Bao-Hua Liu ${ }^{3}$, Jia-Cheng Zheng ${ }^{4}$, Fu-Shuang Dong ${ }^{1}$, \\ Zhi-Fang Liu ${ }^{5}$, Zhi-Yu Wen ${ }^{1}$, Fan Yang ${ }^{1}$, Hai-Bo Wang ${ }^{1}$, Zhao-Shi $\mathrm{Xu}^{6}{ }^{6}{ }^{\circledR}$, He Zhao ${ }^{1, *}$ and \\ Yong-Wei Liu ${ }^{1, *}$ \\ 1 Institute of Genetics and Physiology, Hebei Academy of Agriculture and Forestry Sciences/Plant Genetic \\ Engineering Center of Hebei Province, Shijiazhuang 050051, China; zhoushuobio@163.com (S.Z.); \\ dongfushuang@126.com (F.-S.D.); wzy1800@126.com (Z.-Y.W.); yangfan21st@163.com (F.Y.); \\ nkywanghb@163.com (H.-B.W.) \\ 2 College of Agronomy, Northwest A\&F University, Yangling 712100, China; zhengweijun@nwafu.edu.cn \\ 3 Handan Academy of Agricultural Sciences, Handan 056001, China; hm4589@163.com \\ 4 College of Agronomy, Anhui Science and Technology University, Fengyang, Chuzhou 239000, China; \\ zhengiiachengx2016@126.com \\ $5 \quad$ Hebei Seed Station, Shijiazhuang 050031, China; happysky961@163.com \\ 6 Institute of Crop Sciences, Chinese Academy of Agricultural Sciences (CAAS)/National Key Facility for Crop \\ Gene Resources and Genetic Improvement, Key Laboratory of Biology and Genetic Improvement of Triticeae \\ Crops, Ministry of Agriculture, Beijing 100081, China; xuzhaoshi@caas.cn \\ * Correspondence: hezhao311@163.com (H.Z.); liuywmail@126.com (Y.-W.L.) \\ + These authors contributed equally to this work.
}

Received: 13 October 2019; Accepted: 11 November 2019; Published: 14 November 2019

\begin{abstract}
The WRKY transcription factor superfamily is known to participate in plant growth and stress response. However, the role of this family in wheat (Triticum aestivum L.) is largely unknown. Here, a salt-induced gene TaWRKY13 was identified in an RNA-Seq data set from salt-treated wheat. The results of RT-qPCR analysis showed that TaWRKY13 was significantly induced in NaCl-treated wheat and reached an expression level of about 22-fold of the untreated wheat. Then, a further functional identification was performed in both Arabidopsis thaliana and Oryza sativa L. Subcellular localization analysis indicated that TaWRKY13 is a nuclear-localized protein. Moreover, various stress-related regulatory elements were predicted in the promoter. Expression pattern analysis revealed that TaWRKY13 can also be induced by polyethylene glycol (PEG), exogenous abscisic acid (ABA), and cold stress. After $\mathrm{NaCl}$ treatment, overexpressed Arabidopsis lines of TaWRKY13 have a longer root and a larger root surface area than the control (Columbia-0). Furthermore, TaWRKY13 overexpression rice lines exhibited salt tolerance compared with the control, as evidenced by increased proline (Pro) and decreased malondialdehyde (MDA) contents under salt treatment. The roots of overexpression lines were also more developed. These results demonstrate that TaWRKY13 plays a positive role in salt stress.
\end{abstract}

Keywords: stress responsive mechanisms; TaWRKY transcription factors; salt tolerance

\section{Introduction}

Unlike animals, plants cannot move when exposed to stress. However, complex signaling network have been established to cope with stress [1]. Under stress, a series of responses are induced to prevent or minimize damage. These are accompanied by many physiological, biochemical and developmental changes [2]. Current research on plant stress response has reached the level of cells and molecules, and combined with genetics, we can explore the stress responsive mechanisms in order to improve plant growth under conditions of stress [3-7]. 
Many genes are induced by stress; the products of these genes both participate in stress response and regulate the expression of related genes involved in signal transduction pathways in order to avoid or reduce tissue damage [8-10]. Signaling via the hormone, liposome, SnRK2 (sucrose non-fermenting 1-related protein kinase 2) [11], MAPK (mitogen activated protein kinase) [12], ROS signal [13] and stomatal [14] pathways are the main networks by which plants respond to salt and drought stress. Plant adaptation to drought and other stresses depends on both the expression of stress-resistant related genes and the regulation of various signal pathways induced by stress [15]. Products of stress-related genes can be divided into two classes: the first class includes ion channel proteins [16], water channel proteins [17], osmotic regulators (sucrose, proline and betaine), synthases [18] and other products that directly function in stress response, while the products of the second type include proteins involved in stress-related signal transmission and regulators of gene expression, such as protein kinases (PKs) and transcription factors (TFs) $[19,20]$.

Transcription factors play a crucial role in regulating the expression of stress-related genes in plants. When abiotic stress occurs, changes in the activity of transcription factors cause changes in the activity of target genes. Transcription factors involved in plant stress response are widely researched, such as the AP2/EREBP TF family [21], MYC/MYB TF family [22], HSE binding TFs [23], NAC TF family [24], and WRKY TF family [25]. Among them, WRKY TFs are extensively found in higher plants including Arabidopsis thaliana, Oryza sativa, Setaria italica, Glycine max, and Triticum aestivum, which indicates that WRKY TFs play a significant role in plant stress tolerance [26-30].

Although a large number of studies have shown that WRKY TFs in plants are mainly involved in disease resistance and defense response, some members of the WRKY TFs are involved in abiotic stress response. TaWRKY1 mediates stomatal movement through an ABA-dependent pathway to improve plant tolerance to drought stress [31]. In addition, TaWRKY10 acts as a positive regulator under drought, salt, cold, and hydrogen peroxide stress conditions and improves the stress tolerance in transgenic tobacco [32]. In Arabidopsis, WRKY proteins are involved in regulating ABA response factors, such as MYB2, DREB1a, DREB2a and Rab18 [33]. The overexpression of ZmWRKY33 in Arabidopsis improved the salt-stress tolerance of transgenic plants [34]. These studies suggested that WRKY TFs play a significant role in plant stress response.

High salt stress is a major obstacle to plant growth and development. High salt conditions lead to increases in reactive oxygen species (ROS), metabolic toxicity, membrane disorganization, the inhibition of photosynthesis, and attenuated nutrient acquisition at different plant growth stages [35]. Recent reports claim that salinity affects about $20 \%$ of all irrigated arable land and is an increasing problem in worldwide agriculture (FAO Cereal Supply and Demand Brief. http://www.fao.org/ worldfoodsituation/csdb/en/).

Since wheat is rich in thiamine, fat, calcium, niacin, starch, protein, iron, riboflavin, minerals, and vitamin A and can provide abundant energy and protein for humans, wheat is regarded as one of the most important crops in the world [36]. However, wheat production is constrained by environmental conditions, such as drought, salinity, waterlogging, and extremes in temperature. Next in importance to drought stress, salinity affects crop yields worldwide. The improvement of stress tolerance in wheat by biotechnology and transgenic technology could contribute to increased production worldwide. However, the huge wheat genome has slowed progress [37,38]. Although many studies have investigated the roles of WRKY transcription factors in response to various stress conditions, the mechanisms underlying their function need further study. Here, RNA-Seq, real-time fluorescence quantification PCR (RT-qPCR), and several databases were used in a study of TaWRKY13. The results demonstrated that overexpression of TaWRKY13 can improve salt tolerance in Arabidopsis and rice. 


\section{Results}

\subsection{Identification and Genome Structure Analysis of WRKYs in Triticum aestivum}

According to the Plant Transcription Factor Database website (http://planttfdb.cbi.pku.edu.cn/ index.php), wheat has 171 TaWRKYs, which are distributed across all chromosomes (1AL, 1BL, 1DL, 2AL, 2AS, 2BS, 2DL, 2DS, 3AL, 3B, 3DL, 4AL, 4AS, 4DS, 5AL, 5BS, 5BL, 5DL, 6AL, 6AS, 6BS, 6DS, 7AL, 7DL). Here, PF03106 was used as a key word to blast WRKYs in wheat on the Phytozome website (https://phytozome.jgi.doe.gov/pz/portal.html). Nucleic acid and amino acid sequences of 100 TaWRKYs that harbor at least one WRKY domain are shown in Supplementary Table S2. Based on the rule that the CDS of TaWRKYs were more than 300 base pairs [30], some TaWRKYs were removed, and then combined with the NCBI database (https://www.ncbi.nlm.nih.gov/pubmed), meaning that 57 TaWRKYs were identified with the annotation gene's name, ID, transcript name and location (Table 1). The location of 57 TaWRKYs on chromosomes was analyzed by using the online website http://mg2c.iask.in/mg2c_v2.0/. From the map, we can see that the locations of TaWRKYs were different on each chromosome; for example, TaWRKY $6,38,50,27,48$, and 57 were located at the end of chromosome 3B (forward or reverse), while TaWRKY70 and TaWRKY 71 were located near the centromere of chromosome 1D. Moreover, the distribution of TaWRKYs on 4A was the combination of both distributions described above (Figure 1). To further explore gene structure differences, a gene structure figure of 56 TaWRKYs is displayed in Figure 2. The TaWRKYs are all different in structure. Most TaWRKYs contain 1 to 5 different exons, which may contain different functional structures, such as zinc finger, leucine, kinase structure, exerting different biological functions. TaWRKY7, 22, 23, $24,33,56$, and 90 do not harbor introns, only containing exons and/or an upstream structure.

Table 1. Annotation of WRKY transcription factors in Triticum aestivum.

\begin{tabular}{|c|c|c|c|}
\hline Name & ID & Transcript Name & Location \\
\hline TaWRKY28 & 31740471 & Traes_1BL_9AFA4B870.1 & ta_iwgsc_1bl_v1_3809885:1110..4753 reverse \\
\hline TaWRKY15 & 31742772 & Traes_5BL_E294922A9.2 & ta_iwgsc_5bl_v1_10867378:4708..6402 forward \\
\hline TaWRKY 6 & 31744736 & Traes_3B_CDA5ADD75.1 & ta_iwgsc_3b_v1_10758590:296..2989 forward \\
\hline TaWRKY 62 & 31745499 & Traes_5DL_C93641E43.1 & ta_iwgsc_5dl_v1_4576731:2459..4427 forward \\
\hline TaWRKY44 & 31746115 & Traes_4AL_2EEECCC4B.1 & ta_iwgsc_4al_v2_7093101:3374...6818 forward \\
\hline TaWRKY74 & 31747511 & Traes_5DL_5C93510D5.1 & ta_iwgsc_5dl_v1_4502975:3368..7661 reverse \\
\hline TaWRKY80 & 31748920 & Traes_6AS_DA75BB1FD.1 & ta_iwgsc_6as_v1_4428654:1..1588 reverse \\
\hline TaWRKY53 & 31752041 & Traes_2DL_F600B5FDF.1 & ta_iwgsc_2dl_v1_9719154:1..728 forward \\
\hline TaWRKY22 & 31752743 & Traes_5AL_6FDB440FB.1 & ta_iwgsc_5al_v1_2705439:4..838 forward \\
\hline TaWRKY45 & 31765470 & Traes_7AL_48C81DE03.1 & ta_iwgsc_7al_v1_4556343:539..3297 forward \\
\hline TaWRKY4 & 31765472 & Traes_7AL_48C81DE031.1 & ta_iwgsc_7al_v1_4556343:3718..6476 reverse \\
\hline TaWRKY35 & 31766778 & Traes_6BL_EEAA2A7E3.1 & ta_iwgsc_6bl_v1_4221964:3..2595 forward \\
\hline TaWRKY79 & 31767242 & Traes_7DL_B09854286.1 & ta_iwgsc_7dl_v1_3393496:18..940 reverse \\
\hline TaWRKY46 & 31768080 & Traes_4DS_FE38A59D0.1 & ta_iwgsc_4ds_v1_2280139:4533..7422 reverse \\
\hline TaWRKY57 & 31782323 & Traes_3B_41047D5E6.2 & ta_iwgsc_3b_v1_10527462:3896..6406 reverse \\
\hline TaWRKY33 & 31785825 & Traes_6DS_8F684013D.1 & ta_iwgsc_6ds_v1_1013038:1..419 reverse \\
\hline TaWRKY12 & 31787421 & Traes_6AL_BA4636569.1 & ta_iwgsc_6al_v1_5754118:409..4140 reverse \\
\hline TaWRKY24 & 31792629 & Traes_4AL_C2A825B6D.1 & ta_iwgsc_4al_v2_3841042:1..253 reverse \\
\hline TaWRKY 63 & 31793891 & Traes_3DL_7456F61A3.1 & ta_iwgsc_3dl_v1_5877113:2..2892 reverse \\
\hline TaWRKY 68 & 31798439 & Traes_2AL_15A7BB684.1 & ta_iwgsc_2al_v1_6374918:10015..11505 forward \\
\hline TaWRKY50 & 31799212 & Traes_3B_F45FCFE62.1 & ta_iwgsc_3b_v1_10625585:4077..6054 forward \\
\hline TaWRKY58 & 31811544 & Traes_5BL_D3C383CF5.1 & ta_iwgsc_5bl_v1_10787947:2038..3881 forward \\
\hline TaWRKY72 & 31818595 & Traes_2BS_F3097F116.1 & ta_iwgsc_2bs_v1_5195103:6587..11319 forward \\
\hline TaWRKY8 & 31823877 & Traes_5DL_2553A6C33.1 & ta_iwgsc_5dl_v1_4566006:8..1007 forward \\
\hline TaWRKY34 & 31829399 & Traes_2AL_409AB7647.1 & ta_iwgsc_2al_v1_6334600:3412..8916 reverse \\
\hline
\end{tabular}


Table 1. Cont.

\begin{tabular}{|c|c|c|c|}
\hline Name & ID & Transcript Name & Location \\
\hline TaWRKY9 & 31836810 & Traes_2DS_F6FBC974C.2 & ta_iwgsc_2ds_v1_5331381:733..3264 reverse \\
\hline TaWRKY52 & 31851405 & Traes_3AL_AB2BAE660.1 & ta_iwgsc_3al_v1_4270257:1..887 reverse \\
\hline TaWRKY3 & 31853252 & Traes_2DL_4F9F8F1F0.1 & ta_iwgsc_2dl_v1_9906833:634..5055 reverse \\
\hline TaWRKY51 & 31854913 & Traes_2AL_434E9F101.1 & ta_iwgsc_2al_v1_6367445:3985..5752 reverse \\
\hline TaWRKY27 & 31865868 & Traes_3B_990298FF5.1 & ta_iwgsc_3b_v1_10750391:1..2331 reverse \\
\hline TaWRKY70 & 31871499 & Traes_1DL_DFE1721E0.1 & ta_iwgsc_1dl_v1_2268423:4679..7857 forward \\
\hline TaWRKY41 & 31872073 & Traes_2DS_AD8820C42.1 & ta_iwgsc_2ds_v1_5376167:6..1016 reverse \\
\hline TaWRKY14 & 31872762 & Traes_5BL_B9DD3E76F.1 & ta_iwgsc_5bl_v1_10924584:9637..13927 forward \\
\hline TaWRKY17 & 31875786 & Traes_5BL_8688F70C9.1 & ta_iwgsc_5bl_v1_10840877:2232..3827 reverse \\
\hline TaWRKY56 & 31876237 & Traes_3AL_DED8A29EC.1 & ta_iwgsc_3al_v1_382150:704..961 reverse \\
\hline TaWRKY78 & 31876678 & Traes_2BS_D435A8999.1 & ta_iwgsc_2bs_v1_5214231:8279..15893 reverse \\
\hline TaWRKY32 & 31888413 & Traes_4AS_70DF607CC.1 & ta_iwgsc_4as_v2_352920:1884..3594 forward \\
\hline TaWRKY16 & 31891223 & Traes_4AL_98B1C762B.2 & ta_iwgsc_4al_v2_7173949:3935..6881 forward \\
\hline TaWRKY48 & 31892659 & Traes_3B_B8BF316B8.2 & ta_iwgsc_3b_v1_10433739:23..1890 reverse \\
\hline TaWRKY71 & 31894510 & Traes_1DL_46428511F.1 & ta_iwgsc_1dl_v1_2235906:1905..4608 forward \\
\hline TaWRKY38 & 31895081 & Traes_3B_D6F86ABC3.2 & ta_iwgsc_3b_v1_10762199:7310..8822 forward \\
\hline TaWRKY55 & 31916438 & Traes_6AS_68775100B.1 & ta_iwgsc_6as_v1_4413209:7948..9254 forward \\
\hline TaWRKY76 & 31917474 & Traes_5DL_32D78D06A.1 & ta_iwgsc_5dl_v1_4501324:900..1439 reverse \\
\hline TaWRKY19 & 31924920 & Traes_2BS_380EC4D1E.1 & ta_iwgsc_2bs_v1_5227909:9257..13033 reverse \\
\hline TaWRKY29 & 31938855 & Traes_1AL_4E924201A.1 & ta_iwgsc_1al_v2_3969710:4988..6878 reverse \\
\hline TaWRKY10 & 31942345 & Traes_2DL_362A1F535.1 & ta_iwgsc_2dl_v1_9707610:58..446 forward \\
\hline TaWRKY36 & 31942939 & Traes_3AL_140B829CB.2 & ta_iwgsc_3al_v1_4308486:3673..5708 reverse \\
\hline TaWRKY2 & 31951792 & Traes_5BL_17A712C94.1 & ta_iwgsc_5bl_v1_10916210:5033..9621 forward \\
\hline TaWRKY13 & 31962353 & Traes_2AS_6269D889E.1 & ta_iwgsc_2as_v1_5205891:13214..14843 reverse \\
\hline TaWRKY1 & 31966248 & Traes_5BL_AEF9FE805.1 & ta_iwgsc_5bl_v1_10827243:3081..6424 reverse \\
\hline TaWRKY49 & 31968771 & Traes_3DL_2551BF2C1.1 & ta_iwgsc_3dl_v1_6811598:1..1035 reverse \\
\hline TaWRKY23 & 31977027 & Traes_3AL_4769A72F1.1 & ta_iwgsc_3al_v1_805190:2..774 reverse \\
\hline TaWRKY75 & 31987126 & Traes_1AL_0404BC790.1 & ta_iwgsc_1al_v2_3912777:3..1909 forward \\
\hline TaWRKY5 & 31988149 & Traes_5AL_7164FEAC3.1 & ta_iwgsc_5al_v1_2204788:3..342 forward \\
\hline TaWRKY 64 & 32002393 & Traes_4AS_0DA136E0E.1 & ta_iwgsc_4as_v2_5962726:2807..4440 forward \\
\hline TaWRKY90 & 32002429 & Traes_3AL_1B73D2C12.1 & ta_iwgsc_3al_v1_4248344:659..1678 forward \\
\hline TaWRKY61 & 32024774 & Traes_5BS_C46781248.1 & ta_iwgsc_5bs_v1_2248873:15934..19692 reverse \\
\hline
\end{tabular}

Annotations were according to Phytozome (https://phytozome.jgi.doe.gov/pz/portal.html), PlantTFDB (http:// planttfdb.cbi.pku.edu.cn/index.php and NCBI (https://www.ncbi.nlm.nih.gov/pubmed). 

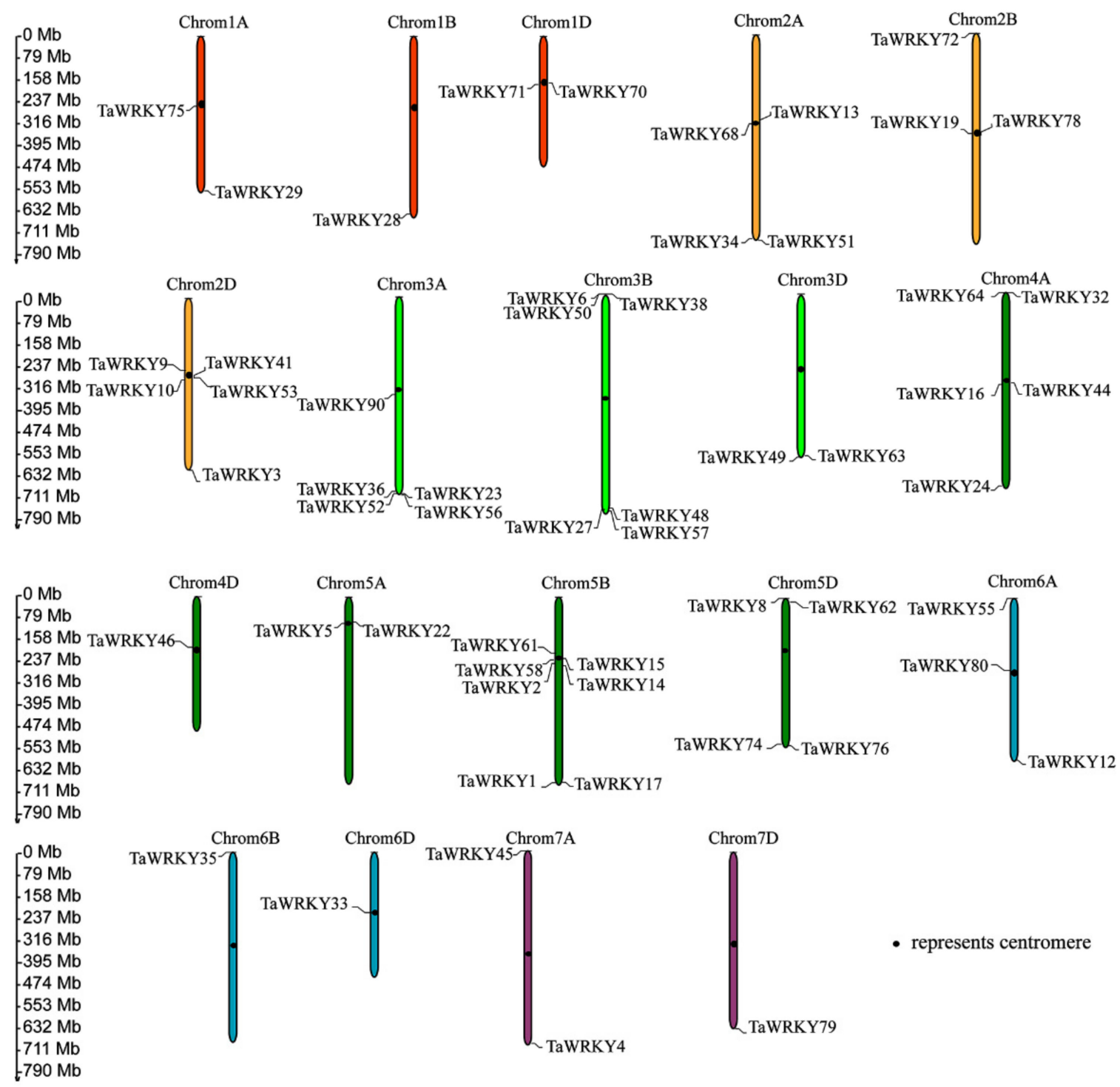

Figure 1. Chromosome location of TaWRKYs listed in Table 1. 


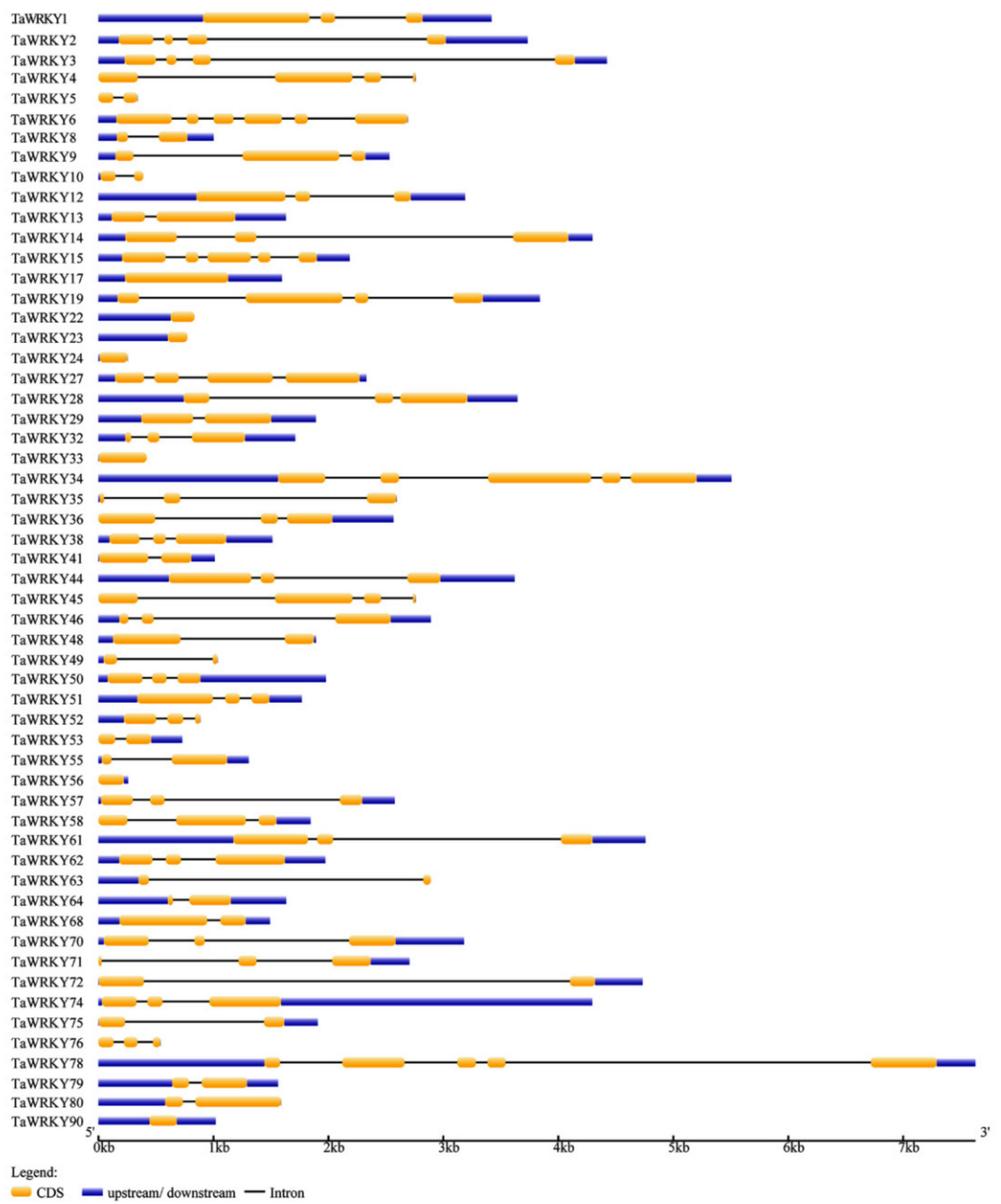

Figure 2. Gene structure analysis of TaWRKYs. Segments in yellow represent CDS, blue indicates upstream/downstream, and black lines represent introns.

\subsection{Identification and Biological Analysis of TaWRKY13}

To find wheat stress-responsive genes under salt stress, the roots of three-leaf wheat seedlings were immersed in $150 \mathrm{mM} \mathrm{NaCl}$ solution for $1 \mathrm{~h}$. Control_Leaf represents the leaf tissue without $\mathrm{NaCl}$ treatment, $\mathrm{NaCl} \_$Leaf represents the leaf tissue treated as per the above description; each treatment involved two independent replicates which were then sampled for RNA-seq (Supplementary Table S1). Twelve TaWRKYs (TaWRKY4, 9, 12, 13, 15, 22, 29, 33, 34, 44, 53, and 70) were selected based on the rule $\log _{2}\left(\mathrm{NaCl} \_L e a f / C o n t r o l \_L e a f\right)>2$. As shown in Figure 3, TaWRKY13 gave the highest relative expression in response to salt stress, peaking at more than 20-fold at $1 \mathrm{~h}$. TaWRKY13 (ID: 31962353, Traes_2AS_6269D889E.1) was selected for further investigation. TaWRKY13 contained a 975 bp open reading frame (ORF) encoding 324 amino acids; the molecular weight of the protein was $81.02 \mathrm{kDa}$ with pI 4.99 (https://web.expasy.org/protparam/). The predicted amino acid sequence showed that TaWRKY13 only harbored one WRKY domain with a highly conserved WRKYGQK motif and a CX4-5CX22-23HXH zinc-finger motif. 

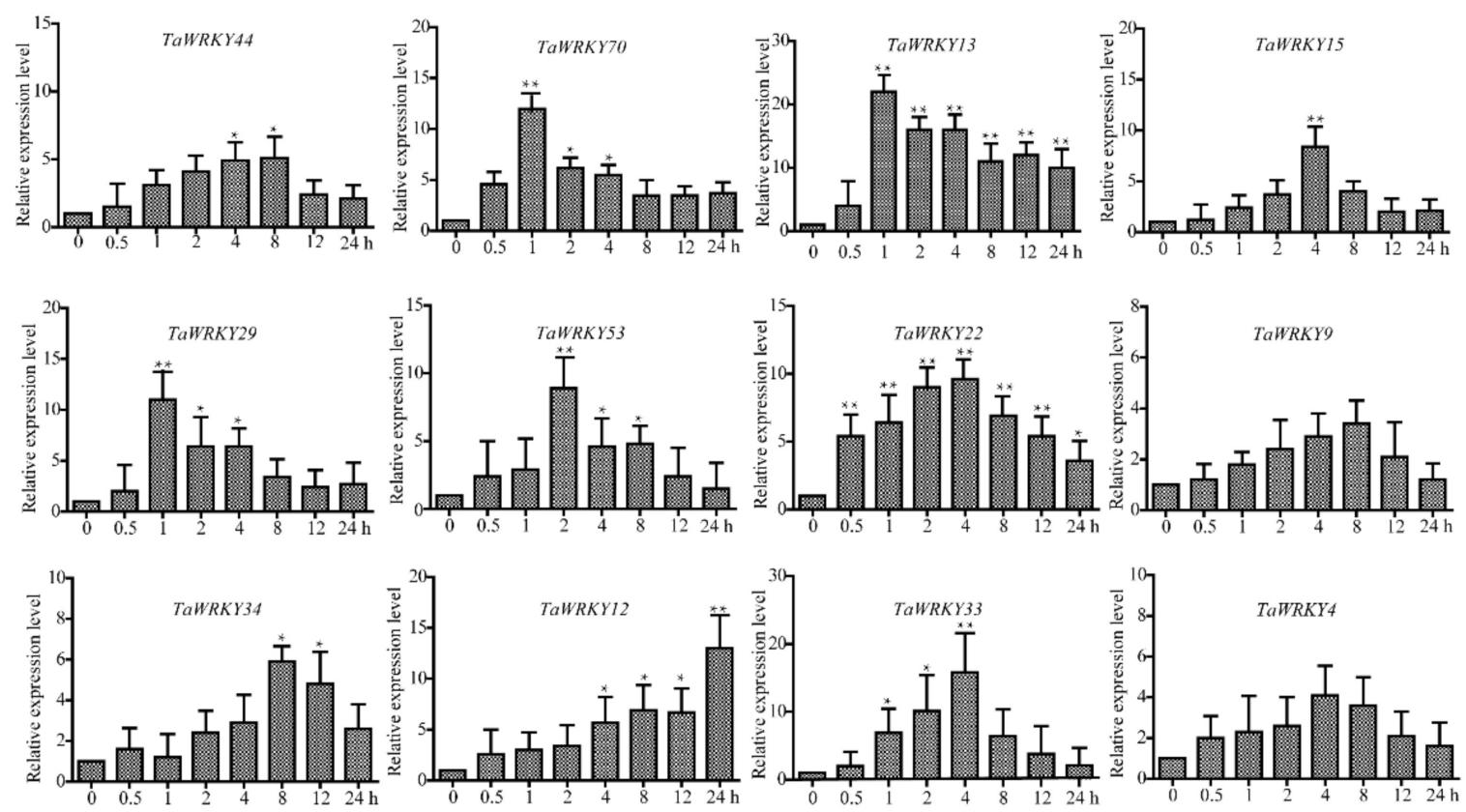

Figure 3. Real-time fluorescence quantification PCR of 12 TaWRKYs under salt treatment. The expression level of TaActin was used as a loading control. The data represent the means \pm SD of three biological replications. The ANOVA demonstrated significant differences $\left({ }^{*} p<0.05,{ }^{* *} p<0.01\right)$.

\subsection{Phylogenetic Analysis of AtWRKYs, OsWRKYs and TaWRKYs}

Phylogenetic analysis is a useful method that can provide some clues to the possible functions of predicted or analyzed target genes. It would be useful to know the homologs of Triticum aestivum WRKYs (TaWRKYs), especially TaWRKY13, with WRKYs of Arabidopsis thaliana (AtWRKYs) and WRKYs of Oryza sativa (OsWRKYs) with reference to previous results. A phylogenic tree was constructed by the neighbor-joining method [39] to investigate the evolutionary relationships between AtWRKYs, OsWRKYs and TaWRKYs. There are 398 WRKYs for phylogenetic analysis (90 AtWRKYs, 128 OsWRKYs and 171 TaWRKYs) (Figure 4). According to Figure 4, AtWRKYs, OsWRKYs and TaWRKYs were scattered across different branches of the phylogenic tree, and all WRKYs were divided into three broad categories; among them, there were more WRKYs in groups I and II than in group III. TaWRKY13 (ID: Traes_2AS_6269D889E.1) and AtWRKY13 (ID: AT4G39410) were in group II, and OsWRKY13 (ID: LOC-Os01g546600) belonged to group I. The results of phylogenetic analysis preliminarily indicated that TaWRKY13 has a closer homology with AtWRKY13 than OsWRKY13. 


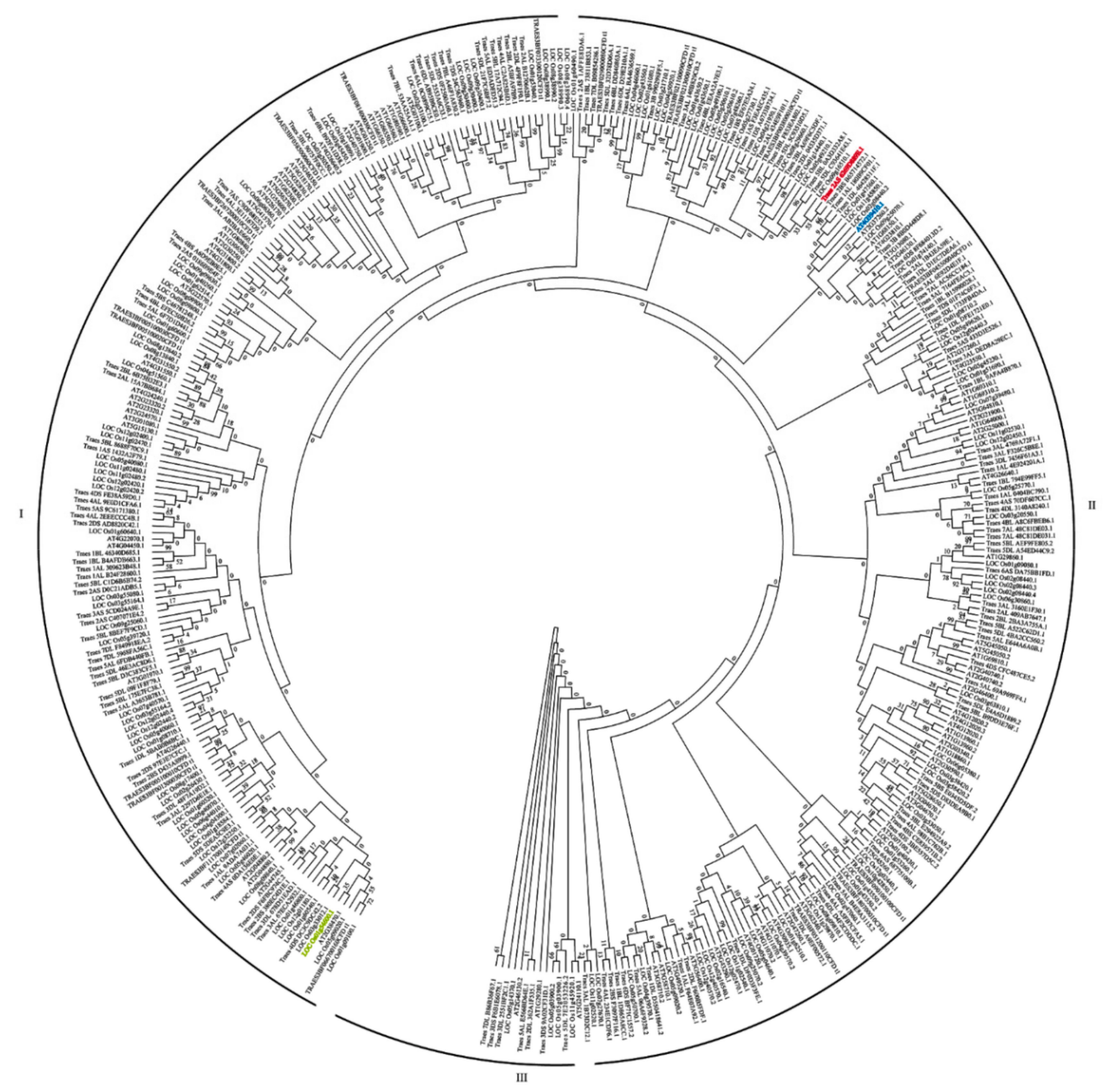

Figure 4. Phylogenetic analysis of AtWRKYs, OsWRKYs and TaWRKYs. The phylogenetic tree was produced using the aligned file with 1000 bootstrap replications in MEGA 6.0. TaWRKY13, AtWRKY13 and OsWRKY13 are highlighted in red, blue and yellow, respectively. The numbers at nodes are bootstrap values, and the length of branches represent evolutionary distance. Number of bootstrap replications: 1000 .

\subsection{TaWRKY13 was Localized in the Nucleus}

To investigate the biological activity of TaWRKY13, the coding sequence fused to the N-terminus of the green fluorescent protein (GFP) was inserted into wheat mesophyll protoplasts by the PEG-mediated method. As the control, 35S::GFP was transformed [40]. The fluorescence of the control GFP was distributed throughout the cells, whereas the fluorescence of 35S::TaWRKY13-GFP was specifically localized in the nucleus (Figure 5). Thus, TaWRKY13 is a nuclear-located protein. 


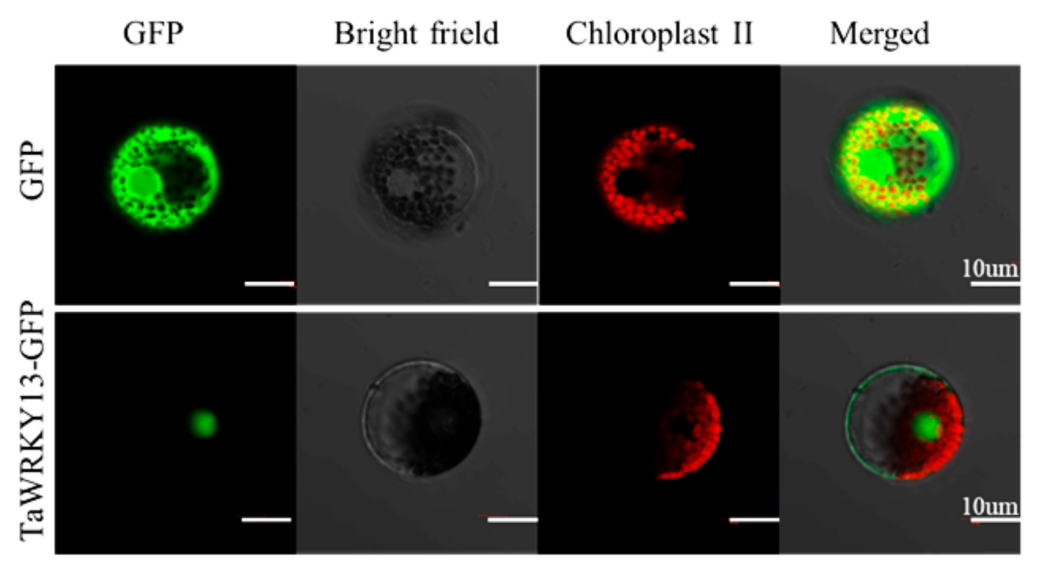

Figure 5. Subcellular localization of TaWRKY13. 35S::GFP and 35S::TaWRKY13-GFP constructs were transformed into wheat mesophyll protoplasts under the control of the Cauliflower Mosaic Virus 35S (CaMV35S) promoter. Wherein, green color represents fluorescence emitted by green fluorescent protein under confocal laser scanning microscope and the red color represents the fluorescence emitted by chloroplasts under confocal laser scanning microscope. Results were observed by a confocal laser scanning microscope (LSM700; CarlZeiss, Oberkochen Germany) after incubation in darkness at $22{ }^{\circ} \mathrm{C}$ for $18-20 \mathrm{~h}$. Scale bars, $10 \mu \mathrm{m}$.

\subsection{Tissue-Specific Expression of TaWRKY13}

Studies of genes with a specific expression in different tissues are necessary to understand the regulatory mechanisms of plant growth and development and the relationship between cell type and function. Here, the promoter sequence of TaWRKY13 was fused to the pCAMBIA1305 vector, which contains a $\beta$-glucuronidase (GUS) reporter gene in the N-terminus (Figure 6). The GUS reporter gene can preliminarily determine the tissue specificity of the gene by observing the tissue location with a blue color after staining [41]. qRT-PCR was used to further verify the relative expression level at the molecular level. TaWRKY13 was expressed in the roots, stems and leaves of $\mathrm{T}_{3}$ generation transgenic Arabidopsis plants under normal and salt-stress conditions, with the relative expression in roots being higher than in leaves and stems. After $\mathrm{NaCl}$ treatment, the expression levels in roots, stems and leaves were significantly increased, indicating that TaWRKY13 might be responsive to salt stress. 

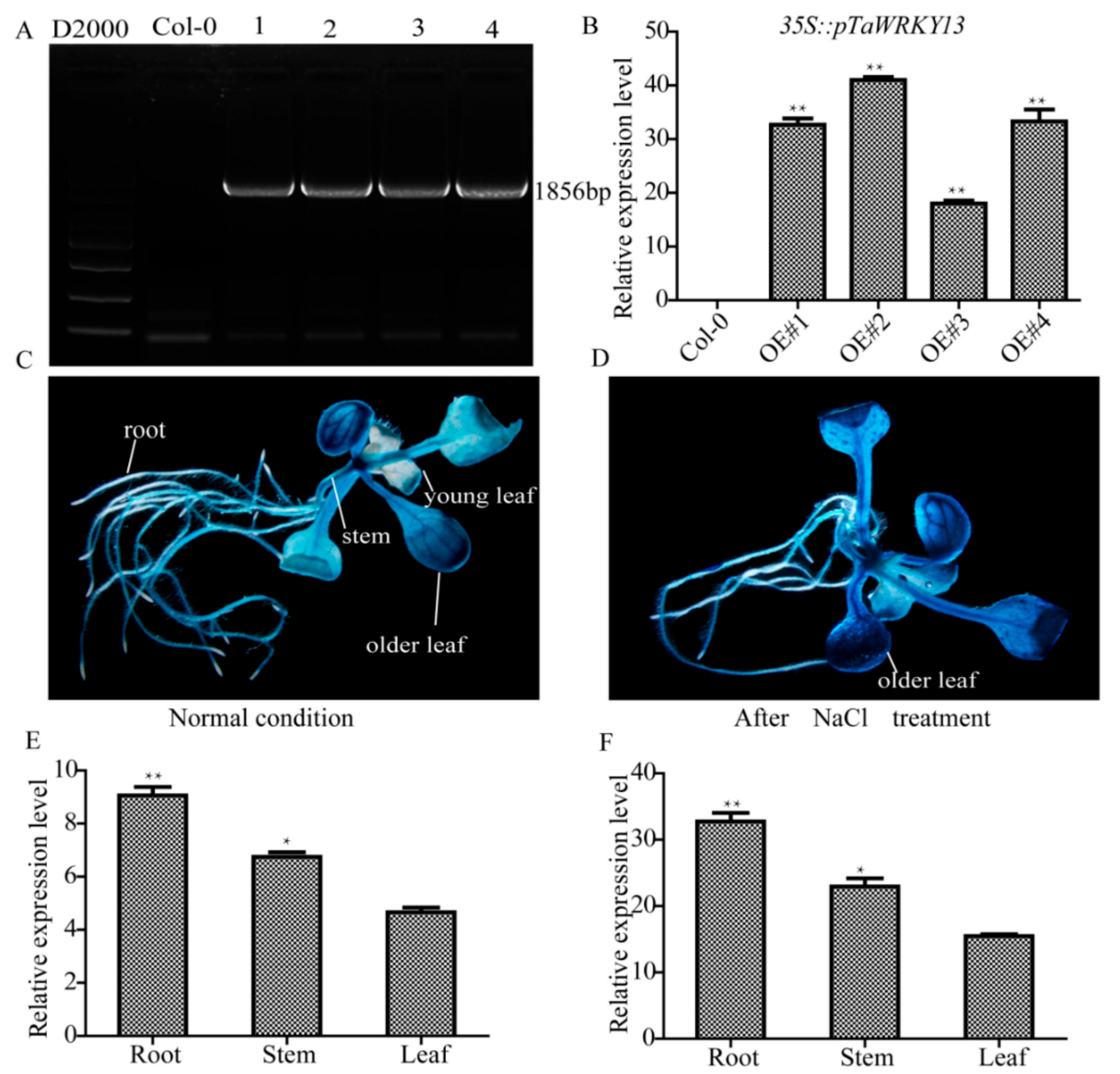

Figure 6. Tissue-specific expression analysis of TaWRKY13. (A) Identification of homozygous lines by agarose gel electrophoresis. (B) Three transgenic lines selected by RT-qPCR. (C) $\beta$-glucuronidase (GUS) staining of transgenic Arabidopsis under normal conditions. (D) GUS staining of transgenic Arabidopsis after $\mathrm{NaCl}$ treatment. (E) qRT-PCR for tissue-specific expression analysis of TaWRKY13 under normal conditions. (F) qRT-PCR for tissue-specific expression analysis of TaWRKY13 after NaCl treatment. All data are means \pm SDs of three independent biological replicates. The ANOVA demonstrated significant differences $\left({ }^{*} p<0.05,{ }^{* *} p<0.01\right)$.

\subsection{TaWRKY13 Is Involved in Various Stress Responses}

WRKY proteins are reported to be involved in various biotic and abiotic stresses [25]. Expression pattern analyses were conducted to determine whether TaWRKY13 was responsive to abiotic stresses. The results indicated that TaWRKY13 participated in salt PEG, ABA and cold-stress responses (Figure 7). For PEG treatment, the relative expression level of TaWRKY13 was rapidly induced at $1 \mathrm{~h}$ after the imposition of PEG stress (Figure 7A). After $\mathrm{NaCl}$ treatment for $1 \mathrm{~h}, \mathrm{TaWRKY} 13$ was highly induced at a maximum level of about 22-fold (Figure 7B). Exogenous ABA and cold stress also significantly affected the expression of TaWRKY13 (Figure 7C,D). The rapid increase in relative expression levels of TaWRKY13 following different stress treatments indicated an important role at the initial stages of stress response. 
A

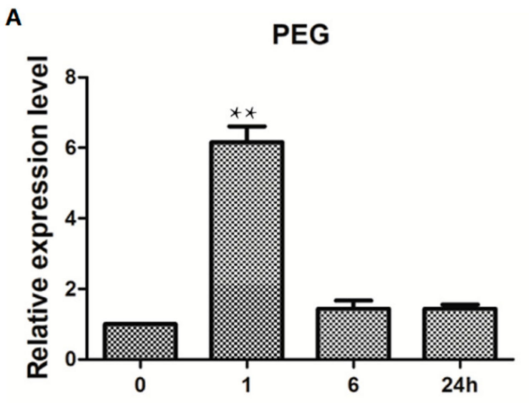

C

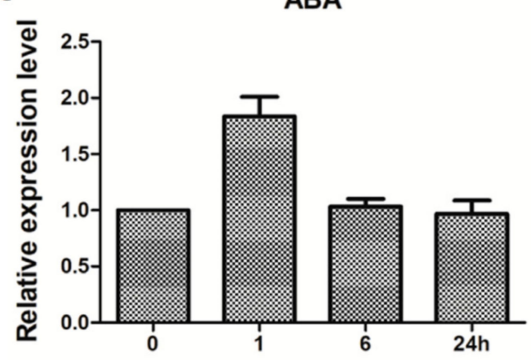

B
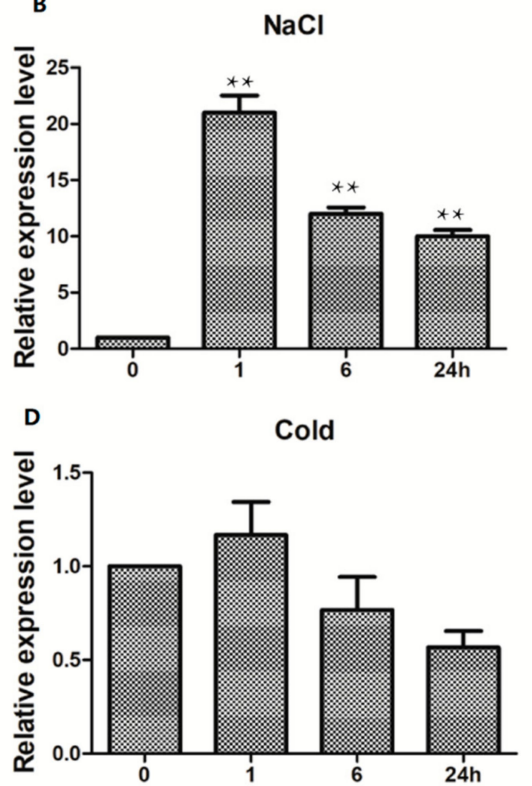

Figure 7. Expression patterns of TaWRKY13 under (A) PEG, (B) $\mathrm{NaCl}$, (C) exogenous abscisic acid (ABA), and (D) cold treatments. The ordinates are relative expression levels (fold) of TaWRKY13 compared to the non-stressed control. The horizontal ordinate is the treatment time, at $0,1,6$ and $24 \mathrm{~h}$. The expression level of TaActin as a loading control. All experiments were repeated three times. Error bars represent standard deviations (SDs). All data are means \pm SDs of three independent biological replicates. The ANOVA demonstrated significant differences $\left({ }^{* *} p<0.01\right)$.

\subsection{Stress-Related Regulatory Elements in the Promoter of TaWRKY13}

The $1.856 \mathrm{~kb}$ promoter region upstream of the TaWRKY13 ATG start codon was isolated to gain an insight into the regulatory mechanism. We searched for putative cis-acting elements in the promoter regions using the database PLACE (http://www.dna.affrc.go.jp/PLACE/). The results are shown in Table 2. Numerous stress-related regulatory elements were present, including a W-BOX, MYB element and TATA-BOX, which take part in the response to both drought and high-salt stress, as well as low-temperature responsive (LTR), ABA-responsive element (ABRE) and GT1, which mainly participate in salt-stress response. Moreover, there were various light, gibberellin, SA (salicylic acid) and high-temperature responsive elements, indicating that TaWRKY13 is involved in abiotic stress response and plant hormone-related signal transduction.

Table 2. Cis-element analysis of the TaWRKY13 promotor.

\begin{tabular}{cccc}
\hline Cis-Element & Target Sequences & Number & Function \\
\hline W-BOX & TTGAC/TGACT & 27 & Drought, high salt responsive elements \\
& TGACY/CTCAY & & Drought, high salt responsive elements \\
MYB & GGATA/WAACCA/TAACARA/ & 20 & Low-temperature, salt responsive elements \\
LTR & TAACAAA/CCWACC/GNGTTR & & ABA-responsive elements \\
ABRE & CCGAC/CCGAAA & 7 & Salt responsive elements \\
TATA-BOX & ACGTGKC & 5 & Drought, cold, high salt responsive elements \\
GTI & TATATAA & 6 & LAAAAA \\
GATA-BOX & GATA & 22 & Light, gibberellin responsive elements \\
WRKY & TAGA & 20 & High temperature responsive elements \\
HSP70A & SCGAYNR(N) $)_{15} \mathrm{HD}$ & 7 & .
\end{tabular}

"Number" corresponds to the number of each type of cis-element in the promoter. 


\subsection{Root System Analysis Indicates That Overexpression Lines Respond to Salt Stress in Arabidopsis}

To explore the mechanism of TaWRKY13 under salt stress, a pCAMBIA1302-TaWRKY13 (35S::TaWRKY13) vector was constructed and transformed into Arabidopsis for root length assay [40]. The results of the identification of homozygotes by agarose gel electrophoresis (AGE) and the selection of three transgenic lines (35S::TaWRKY13\#1, \#2, \#3) by RT-qPCR are available in Supplementary Figure S1. Seedlings of control (Columbia-0) and three $\mathrm{T}_{3}$ generation overexpression lines were first grown on MS (Murashige \& Skoog) medium for one week and then transplanted to MS medium supplemented with various $\mathrm{NaCl}$ concentrations $(0,100,120 \mathrm{mM})$ for salt treatment. As shown in Figure 8, the overexpression lines have an advantage in terms of the main root length and total surface area compared to Col-0 under $\mathrm{NaCl}$ treatment.

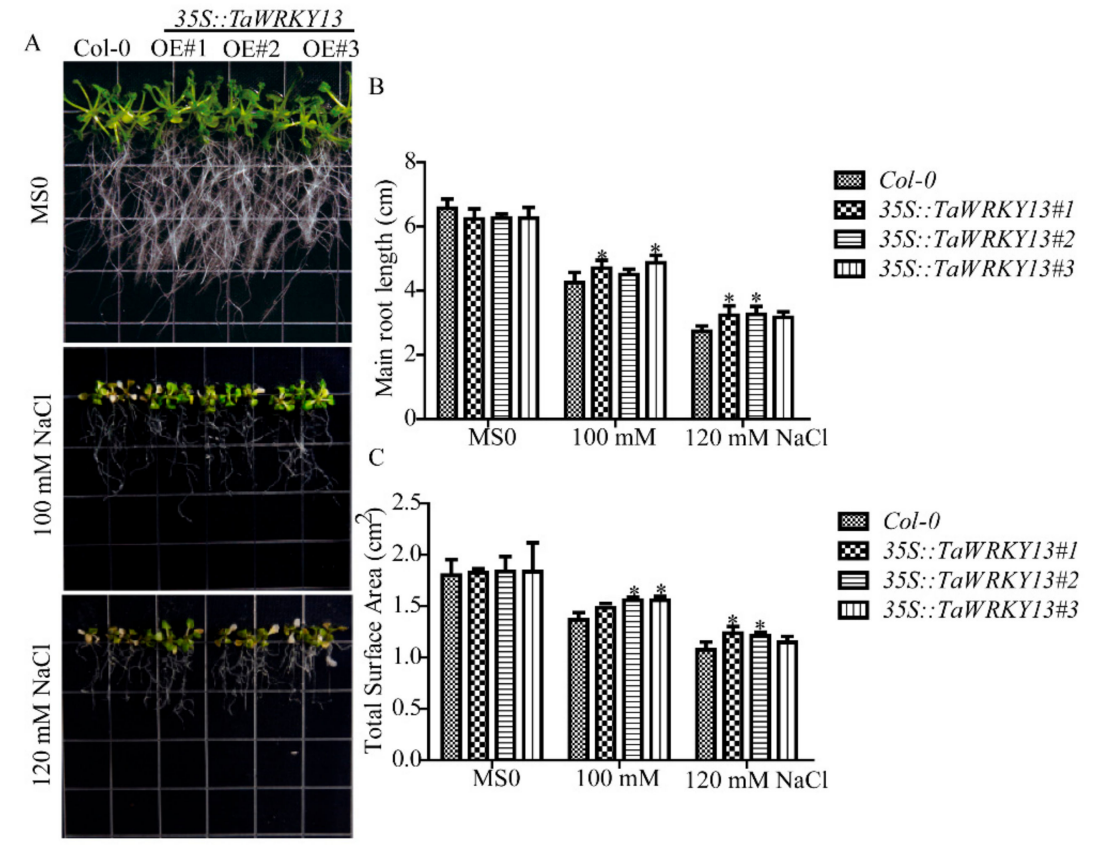

Figure 8. Root length phenotypes of Arabidopsis overexpression lines after $\mathrm{NaCl}$ treatment. (A) Image of the root length phenotype of transgenic lines grown in 0,100 and $120 \mathrm{mM} \mathrm{NaCl}$. (B) Analysis of the main root lengths of transgenic lines under $\mathrm{NaCl}$ treatment. (C) Analysis of total surface areas of transgenic lines under $\mathrm{NaCl}$ treatment. The main root length and total surface area of Arabidopsis roots were measured by the WinRHIZO system. All data are means \pm SDs of three independent biological replicates. The ANOVA demonstrated significant differences $\left({ }^{*} p<0.05\right)$.

\subsection{TaWRKY13 Overexpression Response to Salt Stress in Oryza sativa}

Two-week-old $\mathrm{T}_{3}$ rice lines seedlings of the control (Nipponbare) and three overexpression lines (35S::TaWRKY13\#1, \#2,\#3) were grown hydroponically in untreated control solution or in the same solution supplemented with $150 \mathrm{mM} \mathrm{NaCl}$ to explore the physiological tolerance of TaWRKY13 overexpression rice lines to salt stress [42]. The verification of homozygotes and the selection of three transgenic lines were conducted by AGE and RT-qPCR, respectively (Figure 9A,B). As shown in Figure 9C, before $\mathrm{NaCl}$ treatment, both Nipponbare and the three transgenic lines showed similar growth patterns, with no or little difference in plant height, root length, and proline (Pro) and malondialdehyde (MDA) contents. After 7 days of $\mathrm{NaCl}$ treatment, both Nipponbare and the overexpression lines showed leaf shedding (Figure 9D). Compared with the transgenic lines, Nipponbare plants showed evidence of wilting, water loss and yellowing, whereas the transgenics lines showed less severe symptoms. Meanwhile, the overexpression of TaWRKY13 increased the proline content and decreased MDA content under $\mathrm{NaCl}$ treatment (Figure 9E,F). The root length of Nipponbare was significantly lower than for transgenic plants; the surface areas of transgenic plants were higher than for Nipponbare 
(Figure 9G,H). These results indicated that the overexpression of TaWRKY13 enhanced salt tolerance in rice.

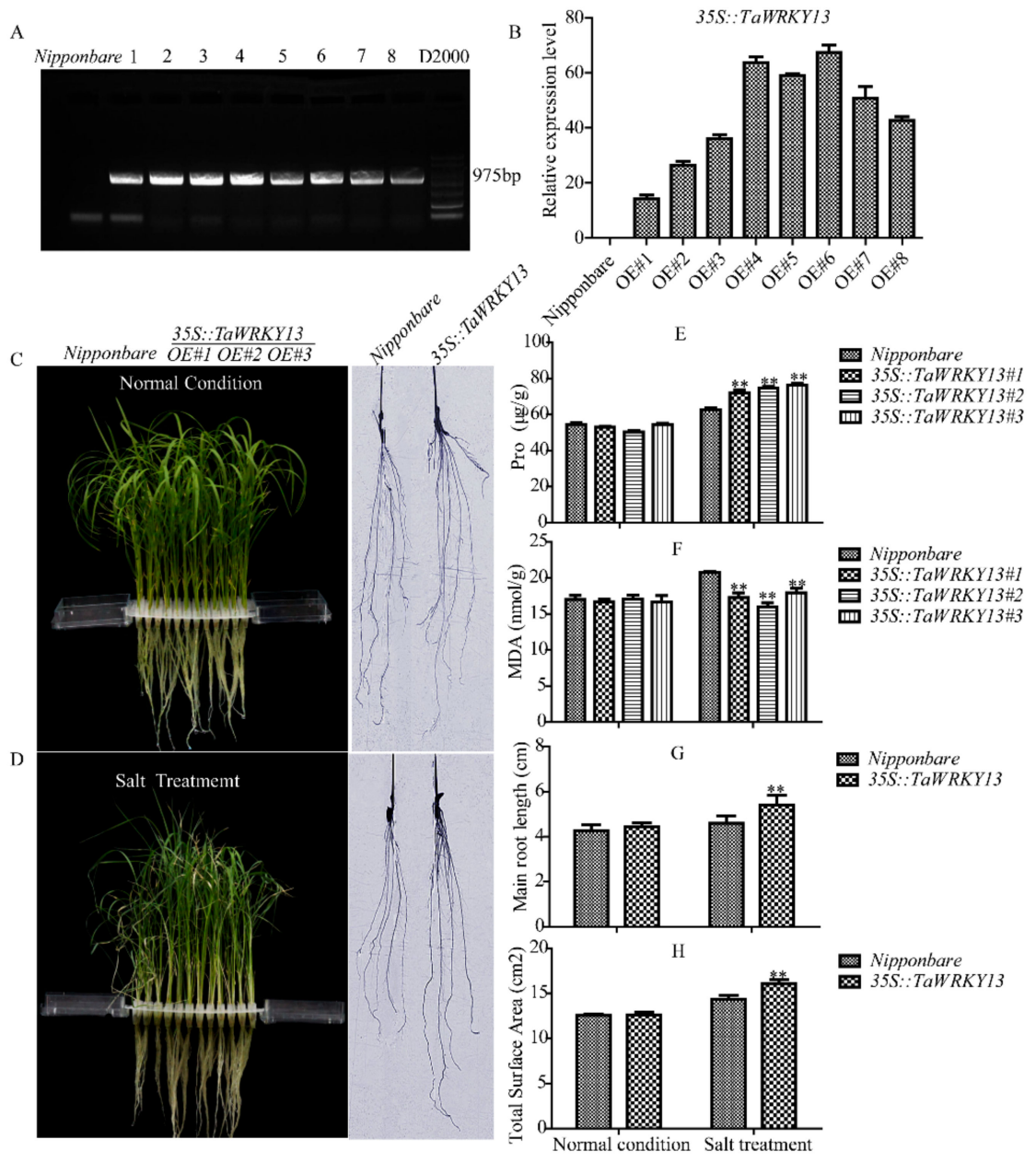

Figure 9. Phenotype identification of TaWRKY13 transgenic rice under NaCl treatment. (A) Confirmation of homozygotes by agarose gel electrphoresis. (B) Selection of three transgenic lines by RT-qPCR. (C) Rice seedlings and root system diagram of Nipponbare and 35S::TaWRKY13 before treatment. (D) Rice seedlings and root system diagram of Nipponbare and 35S::TaWRKY13 after $150 \mathrm{mM} \mathrm{NaCl}$ treatment for 7 days. (E) Proline contents in Nipponbare and 35S:::TaWRKY13 seedlings under normal conditions and $\mathrm{NaCl}$ treatment. (F) Malondialdehyde (MDA) contents in in Nipponbare and 35S::TaWRKY13 rice seedlings under normal growth conditions and $\mathrm{NaCl}$ treatment. (G) Root length measurements of Nipponbare and 35S::TaWRKY13 transformants with and without $\mathrm{NaCl}$ treatment. (H) Total surface areas of Nipponbare and 35S::TaWRKY13 with and without $\mathrm{NaCl}$ treatment. Main root lengths and total surface areas were measured by the WinRHIZO system (Hang xin, Guangzhou, China). All data are means \pm SDs of three independent biological replicates. The ANOVA demonstrated significant differences $(* * p<0.01)$.

\section{Discussion}

Regarded as one group among many important transcription factors in plants, WRKY TFs are represented by 90 members in Arabidopsis and more than 100 in rice [43]. The functions of WRKY TFs have been studied in detail in various plant species since their first discovery.

Since the application of transcriptome sequencing technology, researchers have sequenced the genome of wheat [44,45]. However, owing to the large and complex genome of heterohexaploid wheat, the task has posed many challenges [46]. Recently, transgenic Arabidopsis plants of TaWRKY2 and TaWRKY19 have shown improved stress tolerance, and the overexpression of TaWRKY2 and TaWRKY19 
has exhibited salt, osmotic/dehydration and freezing stress tolerance [47]. More than 160 TaWRKYs were characterized according to their sequence alignment, motif type and phylogenetic relationship analysis by Sezer et al. [48]. Although the WRKY genes associated with stress can be identified by transcriptome sequencing and family analysis, functional identification and mechanism analysis in wheat is limited. Salt stress is one of the most serious stresses that cannot be reversed after damage [49].

Here, on the basis of the previous research, combining RNA-Seq, real-time quantitative PCR (RT-qPCR), and the latest wheat database, TaWRKY13 was isolated from the wheat genome for further study. RNA-Seq was conducted first (Supplementary Table S1); meanwhile, using the wheat database, 57 TaWRKY genes were annotated (Table 1). The results showed that TaWRKYs were differently distributed (number and location) on wheat chromosomes (Figure 1). Studies of the genome structure and the phylogenetic analysis of TaWRKY genes were initially difficult, because the wheat genome was too complex for statistical analysis; there were 171 TaWRKY genes according to the database (https://phytozome.jgi.doe.gov/pz/portal.html). Based on the rule that the CDS of TaWRKYs were more than 300 base pairs, we removed redundant TaWRKY genes and, combined with the NCBI database (https://www.ncbi.nlm.nih.gov/pubmed), 56 TaWRKY genes were selected for the analysis of the gene structure (Figure 2). Major TaWRKY genes harbored different CDS and binding motifs responsible for special function; for example, TaWRKY1 contained an N-terminal CUT domain and a C-terminal NL domain [30]. To further explore TaWRKY genes that respond to salt stress, 12 TaWRKY genes were chosen for verification by qRT-PCR (Figure 3). All 12 genes were up-regulated under salt stress, and TaWRKY13 was chosen for further study due to its higher expression level under salt treatment. Phylogenetic analysis demonstrated that TaWRKY genes have different evolutionary relationships and homologies to WRKYs in Arabidopsis and rice (Figure 4); compared to OsWRKY13, AtWRKY13 was closer to TaWRKY13, possibly indicating similar biological functions [50]. For OsWRKY13, the non-conservation of evolution may provide a basis for the subsequent functional identification of TaWRKY13 in rice, in that the influence of rice itself in OsWRKY13 was eliminated. Subcellular localization showed that TaWRKY13 is a nuclear protein (Figure 5) which may mainly be involved in nuclear signal transduction [51,52]. Although many cotton (Gossypium hirsutum) WRKY genes were expressed at low levels during development, a few GhWRKYs expressed highly in specific tissues such as roots, stems, leaves and embryos [53]. Our results showed that TaWRKY13 was expressed in roots, stems and leaves in transgenic lines, the relative expression level of roots was higher than stems and leaves in transgenic lines, and under salt-stress conditions, the relative expression level was double that of the normal condition (Figure 6).

An increasing number of studies have shown that WRKY TFs play important roles in abiotic stress response; for instance, the overexpression of GmWRKY21 improved cold tolerance in Arabidopsis, because of the regulation of DREB2A and STZ/Zat10. GmWRKY54 conferred salt and drought tolerance; GmWRKY13, which was insensitive to ABA (abscisic acid) but markedly sensitive to salt and mannitol, may function in both lateral root development and the abiotic stress response [54]. Expression pattern analyses revealed that TaWRKY13 was induced significantly by PEG, salt, low-temperature and ABA (Figure 7). Compared with PEG, low-temperature, and ABA stress, TaWRKY13 achieved the highest relative expression level under salt treatment, which was in accordance with the following root length assay in Arabidopsis and the rice resistance assay. Products of WRKY TFs bind to specific cis-regulatory sequences such as the W-BOX in the promoter to induce the expression of downstream target genes [55]. Many regulatory cis-elements that are responsive to drought (W-BOX, MYB and TATA-BOX), high salt (LTR, ABRE and GT1), SA (salicylic acid, WRKY) and cold were recognized in the TaWRKY13 promoter, showing that TaWRKY13 is capable of responding to stress (Table 2). WRKY13 participated in various physiological processes; for example, a weaker stem phenotype, reduced sclerenchyma development, and altered lignin synthesis were observed in an AtWRKY13 mutant, showing that it functioned in stem development [56]. When AtWRKY13 was disturbed under short-day conditions, AtWRKY13 promoted flowering [57]. Furthermore, WRKY13 was also involved in the cross talk between abiotic and biotic stress signaling pathways, and OsWRKY13 displayed selective binding to different cis-elements to 
regulate various stress [58]. In this study, a root length assay of overexpression lines was conducted in Arabidopsis for an analysis of the stress tolerance of TaWRKY13; overexpression lines had longer root lengths and a higher total root area than Col-0 (Figure 8A-C). Additionally, the overexpression of TaWRKY13 enhanced salt tolerance in transgenic rice (Figure 9). Under $\mathrm{NaCl}$ treatment, the transgenic lines of TaWRKY13 grew vigorously, whereas Nipponbare seedlings were more wilted and yellow (Figure 9D); the transgenic lines also had higher proline (Pro) and reduced malondialdehyde (MDA) contents (Figures $8 \mathrm{~F}$ and $9 \mathrm{E}$ ) under $\mathrm{NaCl}$ treatment. In addition, the roots of transgenic lines were longer and more developed than Nipponbare (Figure 9G,H). These results all showed that TaWRKY13 was responsive to salt stress, in agreement with data from other species [54,56,58]. In accordance with the present study, the results suggested that TaWRKY13 has a potential role in improving salt tolerance in wheat. These results are only preliminary in exploring the putative role of TaWRKY13 in salt tolerance; more researches about the role and regulation mechanism of TaWRKY13 are still needed in wheat. For instance, based on the above findings, TaWRKY13 was transformed into wheat for functional verification and mechanism analysis to further improve the role of TaWRKY13 in wheat stress tolerance pathways.

\section{Materials and Methods}

\subsection{De Novo Transcriptome Sequencing of Salt-Treated Wheat}

Wheat (Triticum aestivum L. cultivar Jinhe 9123, from the Institute of Genetics and Physiology, Hebei Academy of Agriculture and Forestry Sciences, Shijiazhuang, China) was cultivated in a $10 \mathrm{~cm} \times 10 \mathrm{~cm}$ pot (vermiculite:soil, 1:3) supplemented with Hoagland's liquid medium at $22^{\circ} \mathrm{C}$ under a $16 \mathrm{hlight} / 8 \mathrm{~h}$ darkness photoperiod for 10 days. When the wheat seedlings were at the three-leaf stage, the pots were immersed in $150 \mathrm{mM} \mathrm{NaCl}$ solution and water (control) for $1 \mathrm{~h}$, respectively [30], prior to the sampling of $0.1 \mathrm{~g}$ fresh leaf tissue. Samples were submerged immediately in liquid nitrogen and stored at $-80^{\circ} \mathrm{C}$ for RNA-Seq. The experiment was performed in three independent replications. In Supplementary Table S1, Control_Leaf means a sample without $\mathrm{NaCl}$ treatment, and $\mathrm{NaCl}$ LLeaf means a sample with salt treatment; each treatment involved three independent replicates, which were then sampled for RNA-Seq. Data are shown in Supplementary Table S1.

\subsection{Identification and Annotation of TaWRKY Response to Salt Stress}

According to the RNA-Seq data, the rule was adopted that the expression level was up-regulated and $\log _{2}(\mathrm{NaCl}$ _treat/Control_treat) $>2$ to select TaWRKYs which responded to salt stress. Several databases-NCBI (https://www.ncbi.nlm.nih.gov/pubmed) PlantTFDB (http://planttfdb.cbi.pku.edu. $\mathrm{cn} /$ ) and Phytozome (https://phytozome.jgi.doe.gov/pz/portal.html)---were used to annotate the gene name, ID, transcript name and localization.

\subsection{Structure Analysis and Phylogenetic Analysis of TaWRKYs}

According to the information listed in Table 1, the chromosome location of TaWRKYs was analyzed by using the online website http://mg2c.iask.in/mg2c_v2.0/. For gene structure analysis, the data of TaWRKYs that were identified in Section 4.2 were uploaded to GSDS (http://gsds.cbi.pku.edu.cn/) to obtain the map of the TaWRKYs' structure. For phylogenetic analysis, a tree of WRKYs from wheat, rice and Arabidopsis was constructed using the neighbor-joining method in MEGA 6.0 with 1000 bootstrap replications [39]. Data for gene structure and the phylogenetic tree analysis were downloaded from PlantTFDB (http://planttfdb.cbi.pku.edu.cn/) and are shown in Supplementary Tables S2 and S3.

\subsection{RNA Extraction of Stress Treatments and RT-qPCR Analyses}

Wheat seeds were sown as previously described; vermiculite and soil were removed by water after being grown for 10 days, and the fresh leaf tissue of three-leaf-stage wheat seedlings were used 
for the RNA extraction of different stress treatments. For the identification of TaWRKY responses to salt stress, the seedlings roots were immersed in $150 \mathrm{mM} \mathrm{NaCl}$ solution, and $0.1 \mathrm{~g}$ of fresh leaf tissue was sampled at different times $(0,0.5,1,2,4,8,12$ and $24 \mathrm{~h})$. For the expression pattern analyses, the roots of wheat seedlings were immersed in 10\% PEG6000, $150 \mathrm{mM} \mathrm{NaCl}$ and $100 \mu \mathrm{mol} \cdot \mathrm{L}^{-1} \mathrm{ABA}$ solutions. Wheat seedlings for cold treatment were placed in a $10 \mathrm{~h}$ light $/ 14 \mathrm{~h}$ darkness, $4 / 2{ }^{\circ} \mathrm{C}$ chamber and sampled at different periods $(0,1,6$ and $24 \mathrm{~h})[30,59,60]$. For specific tissue expression assays, $\mathrm{T}_{3}$ generation transgenic Arabidopsis (35S::pTaWRKY13) plants were surface-sterilized with $10 \%$ Chloros and washed three times with sterile water. Sterilized seeds were sown on MS (Murashige \& Skoog) medium, vernalized in darkness for $3-4$ days at $4{ }^{\circ} \mathrm{C}$, then grown in a chamber at $22{ }^{\circ} \mathrm{C}$ and $75 \%$ humidity under a $16 \mathrm{~h}$ light $/ 8 \mathrm{~h}$ darkness photoperiod for one week. The seedings were transplanted to soil (vermiculite:soil, 1:3), $0.1 \mathrm{~g}$ fresh roots, stems and leaves tissue of 10-day-old transgenic Arabidopsis seedlings with or without $150 \mathrm{mM} \mathrm{NaCl}$ treatment were sampled for RNA the extraction of different tissues [56].

All samples after collection were submerged immediately in liquid nitrogen and stored at $-80^{\circ} \mathrm{C}$ for RNA extraction using an RNA prep plant kit (TIANGEN, Beijing, China); cDNA was synthesized using a Prime Script First-Strand cDNA Synthesis Kit (TransGen, Beijing, China) following the manufacturer's instructions. RT-qPCR was performed with Super Real PreMix Plus (TransGen, Beijing, China) on an ABI Prism 7500 system (Applied Biosystems, Foster city, CA, USA). Specific primers for TaActin, AtActin and TaWRKY4, 9, 12, 13, 15, 22, 19, 33, 34, 44, 53 and 70 for RT-qPCR are listed in Supplementary Table S4. Three biological replicates were used for RT-qPCR analysis, and the $2^{-\Delta \Delta C t}$ method was used for quantification.

\subsection{Gene Isolation and Subcellular Localization}

The ORF (open reading frame) of TaWRKY13 was amplified by PCR with specific primers from wheat cDNA (cultivar Jinhe 9123). The PCR product was fused into pZeroBack vector (TIANGEN, Beijing, China) and sequenced for further study. The correct sequencing plasmids were treated as templates, the segment with restriction sites was amplified by specific primers, and the PCR product was inserted into the N-terminus of the green fluorescent protein (GFP) containing the CaMV35S promoter for subcellular localization; the 35S::GFP vector was used as the control. Both 35S::GFP and 35S::TaWRKY13-GFP were transferred into wheat mesophyll protoplasts by the PEG-mediated method [29]. A confocal laser scanning microscope (LSM700; CarlZeiss, Oberkochen, Germany) was used to observe the fluorescence after incubation in darkness at $22{ }^{\circ} \mathrm{C}$ for $18-20 \mathrm{~h}$. All primers are listed in Supplementary Table S4.

\subsection{Tissue-Specific Expression of TaWRKY13 and GUS Staining}

Tissue-specific expression analysis of TaWRKY13 was conducted by two methods. In the first one, the CDS of TaWRKY13 was amplified as described in Section 4.5, then cloned into the pCAMBIA1302 vector; then, the infected inflorescence of Arabidopsis was determined by the Agrobacterium-mediated method [61], grown as described in Section 4.4, until $\mathrm{T}_{3}$ generation transgenic Arabidopsis seeds were obtained. The identification of homozygotes and selection of three transgenic lines were conducted by agarose gel electrophoresis and RT-qPCR, respectively [59]. The transgenic Arabidopsis seedlings with or without $\mathrm{NaCl}(150 \mathrm{mM})$ treatment were used for RT-qPCR as described in Section 4.4. In the second method, promoter fragments of TaWRKY13 ( $p$ TaWRKY13) were obtained from Ensemble Plants (plants.ensembl.org/index.html); the $p$ TaWRKY13 was amplified by PCR with specific primers from wheat cDNA (Jinhe 9123), and the PCR product was fused into PLB vector (TIANGEN, Beijing, China) and sequenced. The fragment of TaWRKY13 promoter was cloned to the pCAMBIA1305 vector harboring a $\beta$-glucuronidase (GUS) tag, obtaining the $\mathrm{T}_{3}$ generation transgenic Arabidopsis seeds as per the previous description. $\mathrm{T}_{3}$ generation transgenic Arabidopsis (35S::pTaWRKY13) seeds were surface-sterilized, sown on MS medium, vernalized, and grown in a chamber at $22{ }^{\circ} \mathrm{C}$ and $75 \%$ humidity under a $16 \mathrm{~h} \mathrm{light/8} \mathrm{h}$ darkness photoperiod for one week as described in Section 4.4. Ten-day-old 
transgenic Arabidopsis seedlings were submerged to $150 \mathrm{mM} \mathrm{NaCl}$ solution for $1 \mathrm{~h}$. After salt treatment, the liquid was drained with filter paper, and the plant material was subjected to GUS staining solution supplemented with 5-bromo-4-chloro-3-indolylb-d-glucuronic acid (X-gluc) for $3 \mathrm{~h}$; 70\% (vol/vol) ethanol was used to remove the chlorophyll following the manufacturer's protocol (Real-Times, Beijing, China) [56]. GUS staining was observed by a Leica microscope (Wetzlar, Germany). Primers are listed in Supplementary Table S4.

\subsection{Cis-Acting Elements in the TaWRKY13 Promoter}

A $1.856 \mathrm{~kb}$ promoter fragment upstream of the ATG start codon of TaWRKY13 was obtained from the Ensemble Plants website (http://plants.ensembl.org/index.html). Cis-acting elements that respond to various stresses in the promoter region were analyzed by PLACE (http://www.dna.affrc.go. jp/PLACE/) [29].

\subsection{Root Growth Assays of TaWRKY13 under Salt Stress in Arabidopsis}

$\mathrm{T}_{3}$ generation transgenic Arabidopsis lines were obtained as previously described (Section 4.6). Seeds of Col-0 and transgenic lines (35S::TaWRKY13\#1, \#2, \#3) were surface-sterilized, sown on MS medium, vernalized, grown in a chamber at $22{ }^{\circ} \mathrm{C}$ and $75 \%$ humidity under a $16 \mathrm{~h}$ light $/ 8 \mathrm{~h}$ darkness photoperiod for one week as described above (Section 4.4). Three ten-day-old Arabidopsis seedlings (Col-0 and transgenic lines) were transferred to MS medium containing different concentrations of $\mathrm{NaCl}(0,100,120 \mathrm{mM})$ for one week [40].

\subsection{Generation of Transgenic Rice and Stress Identification of TaWRKY13 to Salt Tolerance}

Plant expression vector $p$ CAMBIA1305-TaWRKY13 was constructed and transformed to competent cells of EHA105 as previously described [30]. Genetic transformation was conducted by Dr Chuan-Yin $\mathrm{Wu}$ and colleagues at the Institute of Crop Science, Chinese Academy of Agricultural Sciences using the agrobacterium-mediated method, and Nipponbare was used as the control [62]. The selection of three transgenic lines was made by agarose gel electrophoresis and RT-qPCR, respectively, as previously described (Figure S1). $T_{3}$ generation transgenics (35S::TaWRKY13\#1, \#2, \#3) and Nipponbare were used for further study. Rice seeds were treated with $0.7 \%$ hydrogen peroxide for one day for surface sterilization, breaking dormancy and promoting germination, then replaced with $0.7 \%$ hydrogen peroxide with water and germinated at $37^{\circ} \mathrm{C}$ for 3 days (changing the water once a day). When seeds showed white buds, bare seeds were transplanted to 96 -well plates (24 seeds of Nipponbare and $35 S:: T a W R K Y 13 \# 1, \# 2, \# 3$, respectively) and placed in a growth chamber at $28^{\circ} \mathrm{C}$ and a $16 \mathrm{~h} \mathrm{light} / 8 \mathrm{~h}$ darkness photoperiod and 70\% relative humidity for the hydroponic culture. Seedings were cultured in water for one week, then cultured in water supplemented with Hoagland's hydroponic culture solution. The culture solution was replaced every 5 days, and the $\mathrm{pH}$ was set at 5.5 [63]. Three-leaf seedlings were treated. For salt treatment, the 96-well plates growing three-leaf stage seedlings were transferred to YS hydroponic culture solution and a YS hydroponic culture solution supplemented with $150 \mathrm{mM} \mathrm{NaCl}$ for several days until phenotypes appeared [62]. For each salt treatment, there were three independent replicates. Primers are listed in Supplementary Table S4.

\subsection{Measurements of Proline (Pro) and Malondialdehyde (MDA) Contents}

To better understand the function of TaWRKY13 under salt treatment, proline and MDA contents were measured with Pro and MDA assay kits (Comin, Beijing, China) based on the manufacturer's protocols. Main root lengths and total surface areas of Arabidopsis and rice roots were measured by the WinRHIZO system (Hang xin, Guangzhou, China). Measurements were made on all three biological replicates; means \pm SD and statistically significant differences were based on the ANOVA ${ }^{*} p<0.05$, $\left.{ }^{* *} p<0.01\right)$. 


\section{Conclusions}

We identified the salt-induced WRKY gene TaWRKY13 (ID: 31962353) from a wheat RNA-Seq database (https://phytozome.jgi.doe.gov/pz/portal.html) and real-time quantitative PCR (RT-qPCR). TaWRKY13 is a nuclear protein that was expressed in the roots, stems and leaves of transgenic Arabidopsis. TaWRKY13 was responsive to PEG, salt, cold, and exogenous abscisic acid (ABA) treatment. The overexpression of TaWRKY13 was responsive to salt stress in both Arabidopsis and rice, as evidenced by the promotion of root length and the total root surface area. These results provide a basis for further understanding the functions of TaWRKY13 in wheat when subjected to salt stress.

Supplementary Materials: Supplementary materials can be found at http://www.mdpi.com/1422-0067/20/22/5712/s1. Supplementary Table S1: RNA-Seq data of salt treated wheat. Supplementary Table S2: CDS and amino acid sequences of 100 TaWRKYs used for genome structure and phylogenetic analysis. Supplementary Table S3: Amino acid sequences of 90 AtWRKYs, 128 OsWRKYs and 171 TaWRKY used for phylogenetic analysis. Supplementary Table S4: Primers used in this study. Supplementary Figure S1: Overexpression rice lines. A: Identification of homozygotes by agarose gel electrophoresis B: Selection of three transgenic lines by RT-qPCR.

Author Contributions: Y.-W.L. coordinated the project, wrote and reviewed the manuscript; H.Z. conceived and designed the experiments and edited the manuscript; S.Z. performed the experiments; W.-J.Z. performed validation and formal analysis; B.-H.L., J.-C.Z. and F.-S.D. conducted the bioinformatics and performed related experiments; Z.-S.X. provided analytical tools and analyzed the data; Supervision, Z.-Y.W.; Project administration, Z.-F.L. and F.Y.; Funding acquisition H.-B.W. All authors have read and approved the final manuscript.

Funding: This research was financially supported by the National Natural Science Foundation of China (31600216 and 31871611), the HAAFS Agriculture Science and Technology Innovation Project (2019-4-8-1), and the Natural Science Foundation of Hebei Province (C2017301066).

Acknowledgments: We thank Li-Na Ning for critically reading the manuscript.

Conflicts of Interest: The authors declare no conflict of interest.

\section{Abbreviations}

$\begin{array}{ll}\text { ROS } & \text { Reactive oxygen species } \\ \text { SnRK2 } & \text { Sucrose non-fermenting 1-related protein kinase 2 } \\ \text { MAPK } & \text { Mitogen activated protein kinase } \\ \text { PKs } & \text { Protein kinases } \\ \text { TFs } & \text { Transcription factors } \\ \text { CDS } & \text { Coding sequence } \\ \text { ORF } & \text { Open reading frame } \\ \text { SA } & \text { Salicylic acid } \\ \text { ABA } & \text { Abscisic acid } \\ \text { ABRE } & \text { ABA-responsive element } \\ \text { Pro } & \text { Proline } \\ \text { MDA } & \text { Malondialdehyde } \\ \text { PEG } & \text { Polyethylene glycol } \\ \text { GFP } & \text { Green fluorescent protein } \\ \text { RT-qPCR } & \text { Real-time quantitative PCR } \\ \text { LTR } & \text { Low-temperature responsive } \\ \text { GUS } & \text { B-glucuronidase } \\ \text { X-gluc } & \text { 5-bromo-4-chloro-3-indolylb-d-glucuronic acid } \\ \text { AGE } & \text { Agarose gel electrophoresis } \\ \text { MS } & \text { Murashige \& Skoog } \\ \end{array}$

\section{References}

1. Bechtold, U.; Field, B. Molecular mechanisms controlling plant growth during abiotic stress. J. Exp. Bot. 2018, 69, 2753-2758. [CrossRef] [PubMed]

2. Zhu, J.K. Cell signaling under salt, water and cold stresses. Curr. Opin. Plant Biol. 2001, 4, 401-406. [CrossRef] 
3. Bian, S.M.; Jin, D.H.; Li, R.H.; Xie, X.; Gao, G.I.; Sun, W.K.; Li, Y.J.; Zhai, L.L.; Li, X.Y. Genome-Wide Analysis of CCA1-Like Proteins in Soybean and Functional Characterization of GmMYB138a. Int. J. Mol. Sci. 2017, 18, 2040. [CrossRef] [PubMed]

4. Cao, D.; Li, Y.Y.; Liu, B.H.; Kong, F.J.; Tran, L.P. Adaptive Mechanisms of Soybean Grown on Salt-Affected Soils. Land Degrad. Dev. 2017, 29, 1054-1064. [CrossRef]

5. Cao, Z.H.; Zhang, S.Z.; Wang, R.K.; Zhang, R.F.; Hao, Y.J. Genome Wide Analysis of the Apple MYB Transcription Factor Family Allows the Identification of MdoMYB121 Gene Conferring Abiotic Stress Tolerance in Plants. PLoS ONE 2013, 8, e69955.

6. Degregori, J.; Kowalik, T.; Nevins, J.R. Cellular targets for activation by the E2F1 transcription factor include DNA synthesis- and G1/S-regulatory genes. Mol. Cell. Biol. 1995, 15, 4215. [CrossRef]

7. Lei, Y.; Lu, L.; Liu, H.Y.; Li, S.; Xing, F.; Chen, L.L. CRISPR-P: A web tool for synthetic single-guide RNA design of CRISPR-system in plants. Mol. Plant 2014, 7, 1494-1496. [CrossRef]

8. Lang, V.; Palva, E.T. The expression of a Rab-related gene, rab18, is induced by abscisic acid during the cold acclimation process of Arabidopsis thaliana (L.) Heynh. Plant Mol. Biol. Rep. 1992, 20, 951-962. [CrossRef]

9. Levine, A.; Tenhaken, R.; Dixon, R.; Lamb, C. H2O2 from the oxidative burst orchestrates the plant hypersensitive disease resistance response. Cell 1994, 79, 583-593. [CrossRef]

10. Michael, S.; Markus, H.; Baldwin, I.T.; Emmanuel, G. Jasmonoyl-L-isoleucine coordinates metabolic networks required for anthesis and floral attractant emission in wild tobacco (Nicotiana attenuata). Plant Cell 2014, 26, 3964-3983.

11. Kobayashi, Y.; Murata, M.; Minami, H.; Yamamoto, S.; Kagaya, Y.; Hobo, T.; Yamamoto, A.; Hattori, T. Abscisic acid-activated SNRK2 protein kinases function in the gene-regulation pathway of ABA signal transduction by phosphorylating ABA response element-binding factors. Plant J. Cell. Mol. Biol. 2010, 44, 939-949. [CrossRef] [PubMed]

12. Wang, D. Functions of MAPK Signaling Pathways in the Regulation of Toxicity of Environmental Toxicants or Stresses; Springer: Berlin/Heidelberg, Germany, 2019.

13. Yoshinaga, K.; Arimura, S.I.; Niwa, Y.; Tsutsumi, N.; Uchimiya, H.; Kawai-Yamada, M. Mitochondrial Behaviour in the Early Stages of ROS Stress Leading to Cell Death in Arabidopsis thaliana. Ann. Bot. 2005, 96, 337-342. [CrossRef] [PubMed]

14. Zhao, Z.; Zhang, W.; Stanley, B.A.; Assmann, S.M. Functional proteomics of Arabidopsis thaliana guard cells uncovers new stomatal signaling pathways. Plant Cell 2008, 20, 3210-3226. [CrossRef] [PubMed]

15. Peng, Z.; He, S.; Gong, W.; Sun, J.; Pan, Z.; Xu, F.; Lu, Y.; Du, X. Comprehensive analysis of differentially expressed genes and transcriptional regulation induced by salt stress in two contrasting cotton genotypes. BMC Genom. 2014, 15, 760. [CrossRef] [PubMed]

16. Runnels, L.W.; Yue, L.; Clapham, D.E. TRP-PLIK, a bifunctional protein with kinase and ion channel activities. Science 2001, 291, 1043-1047. [CrossRef] [PubMed]

17. Mulders, S.M.; Preston, G.M.; Deen, P.M.; Guggino, W.B.; van Os, C.H.; Agre, P. Water channel properties of major intrinsic protein of lens. J. Biol. Chem. 1995, 270, 9010-9016. [CrossRef] [PubMed]

18. Yovchev, A.G.; Stone, A.K.; Hucl, P.; Scanlon, M.G.; Nickerson, M.T. Polyethylene glycol as an osmotic regulator in dough with reduced salt content. J. Cereal Sci. 2017, 76, 193-198. [CrossRef]

19. Hunter, T. Protein kinases and phosphatases: The yin and yang of protein phosphorylation and signaling. Cell 1995, 80, 225. [CrossRef]

20. Madhunita, B.; Ralf, O. WRKY transcription factors: Jack of many trades in plants. Plant Signal. Behav. 2014, 9, e27700.

21. Xu, Z.S.; Chen, M.; Li, L.C.; Ma, Y.Z. Functions and application of the AP2/ERF transcription factor family in crop improvement. J. Integr. Plant Biol. 2011, 53, 570-585. [CrossRef]

22. Abe, H.; Yamaguchishinozaki, K.; Urao, T.; Iwasaki, T.; Hosokawa, D.; Shinozaki, K. Role of Arabidopsis MYC and MYB homologs in drought- and abscisic acid-regulated gene expression. Plant Cell 1997, 9, 1859-1868. [PubMed]

23. Fiorenza, M.T.; Farkas, T.; Dissing, M.; Kolding, D.; Zimarino, V. Complex expression of murine heat shock transcription factors. Nucleic Acids Res. 1995, 23, 467-474. [CrossRef] [PubMed]

24. Nakashima, K.; Takasaki, H.; Mizoi, J.; Shinozaki, K.; Yamaguchishinozaki, K. NAC transcription factors in plant abiotic stress responses. BBA Gene Regul. Mech. 2012, 1819, 97-103. [CrossRef] [PubMed] 
25. Eulgem, T.; Rushton, P.J.; Robatzek, S.; Somssich, I.E. The WRKY superfamily of plant transcription factors. Trends Plant Sci. 2000, 5, 199-206. [CrossRef]

26. Chen, J.; Nolan, T.; Ye, H.; Zhang, M.; Tong, H.; Xin, P.; Chu, J.; Chu, C.; Li, Z.; Yin, Y. Arabidopsis WRKY46, WRKY54 and WRKY70 Transcription Factors Are Involved in Brassinosteroid-Regulated Plant Growth and Drought Response. Plant Cell 2017, 29, 1425. [CrossRef] [PubMed]

27. Lee, H.; Cha, J.; Choi, C.; Choi, N.; Ji, H.S.; Park, S.R.; Lee, S.; Hwang, D.J. Rice WRKY11 Plays a Role in Pathogen Defense and Drought Tolerance. Rice 2018, 11, 5. [CrossRef]

28. Muthamilarasan, M.; Bonthala, V.S.; Khandelwal, R.; Jaishankar, J.; Shweta, S.; Nawaz, K.; Prasad, M. Global analysis of WRKY transcription factor superfamily in Setariaidentifies potential candidates involved in abiotic stress signaling. Front. Plant Sci. 2015, 6, 910. [CrossRef]

29. Shi, W.Y.; Du, Y.T.; Ma, J.; Min, D.H.; Zhang, X.H. The WRKY transcription factor GmWRKY12 confers drought and salt tolerance in soybean. Int. J. Mol. Sci. 2018, 19, 4087. [CrossRef]

30. He, G.H.; Xu, J.Y.; Wang, Y.X.; Liu, J.M.; Li, P.S.; Chen, M.; Ma, Y.Z.; Xu, Z.S. Drought-responsive WRKY transcription factor genes TaWRKY1 and TaWRKY33 from wheat confer drought and/or heat resistance in Arabidopsis. BMC Plant Biol. 2016, 16, 116. [CrossRef]

31. Ding, W.; Fang, W.; Shi, S.; Zhao, Y.; Li, X.; Xiao, K. Wheat WRKY Type Transcription Factor Gene TaWRKY1 is Essential in Mediating Drought Tolerance Associated with an ABA-Dependent Pathway. Plant Mol. Biol. Rep. 2016, 34, 1111-1126. [CrossRef]

32. Chen, W.; Deng, P.; Chen, L.; Wang, X.; Hui, M.; Wei, H.; Yao, N.; Ying, F.; Chai, R.; Yang, G. A Wheat WRKY Transcription Factor TaWRKY10 Confers Tolerance to Multiple Abiotic Stresses in Transgenic Tobacco. PLOS ONE 2013, 8, e65120.

33. Rushton, D.L.; Tripathi, P.; Rabara, R.C.; Lin, J.; Ringler, P.; Boken, A.K.; Langum, T.J.; Smidt, L.; Boomsma, D.D.; Emme, N.J. WRKY transcription factors: Key components in abscisic acid signalling. Plant Biotech. J. 2011, 10, 2-11. [CrossRef] [PubMed]

34. Li, H.; Gao, Y.; Xu, H.; Dai, Y.; Deng, D.; Chen, J. ZmWRKY33, a WRKY maize transcription factor conferring enhanced salt stress tolerances in Arabidopsis. Plant Growth Regul. 2013, 70, 207-216. [CrossRef]

35. Hasegawa, P.M.; Bressan, R.A.; Zhu, J.K.; Bohnert, H.J. Plant Cellular and Molecular Responses to High Salinity. Annu. Rev. Plant Biol. 2000, 51, 463-499. [CrossRef] [PubMed]

36. Munns, R.; James, R.A.; Läuchli, A. Approaches to increasing the salt tolerance of wheat and other cereals. J. Exp. Bot. 2006, 57, 1025-1043. [CrossRef] [PubMed]

37. Röder, M.S.; Korzun, V.; Wendehake, K.; Plaschke, J.; Tixier, M.; Leroy, P.; Ganal, M.W. A Microsatellite Map of Wheat. Genetics 1998, 149, 2007.

38. Somers, D.; Isaac, P.; Edwards, K. A high-density microsatellite consensus map for bread wheat (Triticum aestivum L.). Theo. Appl. Genet. 2004, 109, 1105-1114. [CrossRef]

39. Tamura, K.; Stecher, G.; Peterson, D.; Filipski, A.; Kumar, S. MEGA6: Molecular evolutionary genetics analysis version 6.0. Mol. Biol. Evol. 2013, 30, 2725-2729. [CrossRef]

40. Du, Y.T.; Zhao, M.J.; Wang, C.T.; Gao, Y.; Wang, Y.X.; Liu, Y.W.; Chen, M.; Chen, J.; Zhou, Y.B.; Xu, Z.S.; et al. Identification and characterization of GmMYB118 responses to drought and salt stress. BMC Plant Biol. 2018, 18, 320. [CrossRef]

41. Martin, T.; Wöhner, R.V.; Hummel, S.; Willmitzer, L.; Frommer, W.B.; Gallagher, S.R. The GUS reporter system as a tool to study plant gene expression. Gus Protocols 1992, 23-43. [CrossRef]

42. James, D.; Borphukan, B.; Fartyal, D.; Ram, B.; Yadav, R.; Singh, J.; Manna, M.; Panditi, V.; Sheri, V.; Achary, M. Concurrent Overexpression of OsGS1;1 and OsGS2 Genes in Transgenic Rice (Oryza sativa L.): Impact on Tolerance to Abiotic Stresses. Front. Plant Sci. 2018, 9, 786. [CrossRef] [PubMed]

43. Ross, C.A.; Liu, Y.; Shen, Q.J. The WRKY Gene Family in Rice (Oryza sativa). J. Integr. Plant Biol. 2010, 49, 827-842. [CrossRef]

44. Garnica, D.P.; Upadhyaya, N.M.; Dodds, P.N.; Rathjen, J.P. Strategies for Wheat Stripe Rust Pathogenicity Identified by Transcriptome Sequencing. PLoS ONE 2013, 8, e67150. [CrossRef] [PubMed]

45. Pingault, L.; Choulet, F.; Alberti, A.; Glover, N.; Wincker, P.; Feuillet, C.; Paux, E. Deep transcriptome sequencing provides new insights into the structural and functional organization of the wheat genome. Genome Biol. 2015, 16, 29. [CrossRef]

46. Feng, Y.; Zhao, Y.; Wang, K.; Yong, C.L.; Xiang, W.; Yin, J. Identification of vernalization responsive genes in the winter wheat cultivar Jing841 by transcriptome sequencing. J. Genet. 2016, 95, 1-8. [CrossRef] 
47. Niu, C.F.; Wei, W.; Zhou, Q.Y.; Tian, A.G.; Hao, Y.J.; Zhang, W.K.; Ma, B.; Lin, Q.; Zhang, Z.B.; Zhang, J.S. Wheat WRKY genes TaWRKY2 and TaWRKY19 regulate abiotic stress tolerance in transgenic Arabidopsis plants. Plant Cell Environ. 2012, 35, 1156-1170. [CrossRef]

48. Sezer, O.; Ebru, D.; Turgay, U. Transcriptome-wide identification of bread wheat WRKY transcription factors in response to drought stress. Mol. Genet. Genom. 2014, 289, 765-781.

49. Kawasaki, S.; Borchert, C.; Deyholos, M.; Hong, W.; Brazille, S.; Kawai, K.; Galbraith, D.; Bohnert, H.J. Gene Expression Profiles during the Initial Phase of Salt Stress in Rice. Plant Cell 2001, 13, 889-905. [CrossRef]

50. Cavallisforza, L.L.; Edwards, A.W. Phylogenetic analysis. Models and estimation procedures. Am. J. Hum. Genet. 1967, 21, 550-570.

51. Chen, J.N.; Yin, Y.H. WRKY Transcription Factors are Involved in Brassinosteroid Signaling and Mediate the Crosstalk Between Plant Growth and Drought Tolerance. Plant Signal. Behav. 2017, 12, e1365212. [CrossRef]

52. Cheng, H. Overexpression of the GmNAC2 Gene, an NAC Transcription Factor, Reduces Abiotic Stress Tolerance in Tobacco. Plant Mol. Biol. Rep. 2013, 31, 435-442.

53. Ling, L.D.; Xiao, H.Z.; Chao, Y.P.; Mei, Z.S.; Heng, L.W.; Shu, L.F.; Shu, X.Y. Genome-wide analysis of the WRKY gene family in cotton. Mol. Genet. Genom. 2014, 289, 1103.

54. Zhou, Q.Y.; Tian, A.G.; Zou, H.F.; Xie, Z.M.; Lei, G.; Huang, J.; Wang, C.M.; Wang, H.W.; Zhang, J.S.; Chen, S.Y. Soybean WRKY-type transcription factor genes, GmWRKY13, GmWRKY21, and GmWRKY54, confer differential tolerance to abiotic stresses in transgenic Arabidopsis plants. Plant Biotech. J. 2010, 6, 486-503. [CrossRef] [PubMed]

55. Yu, Y.; Wang, N.; Hu, R.; Xiang, F. Genome-wide identification of soybean WRKY transcription factors in response to salt stress. Springerplus 2016, 5, 920. [CrossRef]

56. Li, W.; Tian, Z.; Yu, D. WRKY13 acts in stem development in Arabidopsis thaliana. Plant Sci. 2015, 236, $205-213$. [CrossRef]

57. Wei, L.; Wang, H.; Yu, D. The Arabidopsis WRKY transcription factors WRKY12 and WRKY13 oppositely regulate flowering under short-day conditions. Mol. Plant 2016, 9, 1492-1503.

58. Jun, X.; Hongtao, C.; Xianghua, L.; Jinghua, X.; Caiguo, X.; Shiping, W. Rice WRKY13 Regulates Cross Talk between Abiotic and Biotic Stress Signaling Pathways by Selective Binding to Different cis-Elements. Plant Phys. 2013, 163, 1868-1882.

59. Cui, X.Y.; Du, Y.T.; Fu, J.D.; Yu, T.F.; Wang, C.T.; Chen, M.; Chen, J.; Ma, Y.Z.; Xu, Z.S. Wheat CBL-interacting protein kinase 23 positively regulates drought stress and ABA responses. BMC Plant Biol. 2018, 18, 93. [CrossRef]

60. Radkova, M.; Vítámvás, P.; Sasaki, K.; Imai, R. Development- and cold-regulated accumulation of cold shock domain proteins in wheat. Plant Physiol. Bioch. 2014, 77, 44-48. [CrossRef]

61. Bradley, D.; Ratcliffe, O.; Vincent, C.; Carpenter, R.; Coen, E. Inflorescence commitment and architecture in Arabidopsis. Science 1997, 275, 80-83. [CrossRef]

62. Hiei, Y.; Ohta, S.; Komari, T.; Kumashiro, T. Efficient transformation of rice (Oryza sativa L.) mediated by Agrobacterium and sequence analysis of the boundaries of the T-DNA. Plant J. Cell Mol. Biol. 2010, 6, 271-282. [CrossRef] [PubMed]

63. Yoshida, S.; Forno, D.A.; Cock, J.H. Laboratory Manual for Physiological Studies of Rice; CABI: Wallingford, UK, 1971.

(C) 2019 by the authors. Licensee MDPI, Basel, Switzerland. This article is an open access article distributed under the terms and conditions of the Creative Commons Attribution (CC BY) license (http://creativecommons.org/licenses/by/4.0/). 